\title{
The Challenging and Amazing Field of Sports Cardiology
}

\section{Carmen Adamuz ${ }^{1}$ - Domingo Pascual Figal ${ }^{2}$}

Published online: 16 June 2020

(C) Springer Science+Business Media, LLC, part of Springer Nature 2020
Sports cardiology is one of the most challenging fields in clinical cardiology, where both knowledge and experience deal with difficult scenarios in a daily basis. This special issue includes pearls from recognized specialists in sports cardiology aimed to improve the skills of the reader with up-to-date, state-of-the-art reviews on the cardiovascular management of athlete in some of these specific scenarios.

Athletic adaptation is associated with structural changes of the cardiovascular system that frequently overlap those observed in individuals with cardiomyopathies, one of the leading causes of death in young athletes; therefore, how to discriminate athlete's heart from cardiomyopathies remains one of the most intriguing questions in sports cardiology. In the first of the articles, Marc Abuli et al. [1] address this question from a practical perspective, and structured approach focused on key differential diagnostic characteristics found on the 12-leads ECG, echocardiography and CMR of athlete's vs the most common forms of left ventricle cardiomyopathies. Arrhythmogenic cardiomyopathy deserves special attention as competitive sports participation may not only trigger ventricular arrhythmias and cause sudden death in young athletes but also contribute to accelerate the progression of the disease. Initially considered a right ventricle disease, its left ventricle dominant form is increasingly being recognize in athletes despite the difficulties to stablish a clinical suspicious in the cardiac assessment of asymptomatic athletes. Experts from the University of Padova, with Alessandro Zorzi as first author [2], provide an excellent insight on the current approach to the early identification of arrhythmogenic cardiomyopathy, including left

Domingo Pascual Figal

dpascual@um.es

M. Carmen Adamuz

MariaDelCarmen.Adamuz@aspetar.com

1 Sports Medicine Department, Aspetar, Qatar Orthopaedic and Sports Medicine Hospital, P.O. Box 29222, Doha, Qatar

2 Head of Cardiology Department, Hospital Virgen de la Arrixaca, University of Murcia, Centro Nacional de Investigaciones Cardiovasculares (CNIC), Murcia, Spain ventricle dominant forms, which have the potential to modify the natural history of the disease. Risk assessment and eligibility decisions including specific recommendations for leisure time sports participation for those with arrhythmogenic cardiopathy are important part of the appropriate management of athletes that require the expertise of the sports cardiologist.

Another difficult scenario, easily misunderstood, is the diagnosis of myocarditis, which have a wide range of clinical manifestations among which the ventricular arrhythmias and sudden cardiac death may be the first manifestation both in the acute and the chronic phase, linked to the development of myocardial fibrosis. Guided by the expertise of Domenico Corrado and its team [3], the third article of this issue addresses some of the challenges on the diagnosis and management of athletes with myocarditis. Current coronavirus disease 2019 - COVID19 - pandemic provides a new clinical scenario for sports cardiologist. Although the disease is not specifically targeted on this article, subclinical forms of myocardial involvement and chronic myocardial scars common to other forms of myocarditis may raise in our athletic population in coming months, for what this paper may represent a perfect guidance for the clinician in this new COVID19 era.

In all these difficult scenarios, cardiac magnetic resonance (CMR) is now recognized as the most useful tool for the diagnosis, and we have included a beautiful review on this topic. Viviana Maestrini as first author and James Moon as senior author provide an excellent overview of the role of CMR in sports cardiology on the fourth article of this issue [4]. CMR can more precisely assess cardiac structure and function as well as characterize the myocardium, detecting key changes including myocardial scar, diffuse fibrosis and oedema that will otherwise not being identify, providing substantial certainty in many "grey area" cases. CMR has provided to be particularly utile when athletes present with abnormal ECGs or ventricular arrhythmias and apparently normal on inconclusive echocardiographic changes. However, an accurate diagnosis requires that CMR is performed appropriately with appropriate reference ranges for male and particularly for female athletes, and in the context of the for different sporting categories and training regimens, with 
caution to avoid over-interpreting normal findings on the athletic population.

Another relevant question is the influence of age on the cardiovascular adaptation, which is an important modulator of findings and management. We have therefore included specific articles from experienced group addressing the paediatric population and master athletes. The cardiac care of the paediatric athlete is beautifully addressed by Guido Pieles and Renate Oberhoffer with the aim to provide guidance on how to distinguish adaptive, beneficial cardiovascular remodelling from underlying pathology of congenital or inherited cardiovascular disease in children [5]. Differences in presentation, diagnosis and treatment between childhood and adult athletes are highlighted and can educate the reader in the emerging field of paediatric sports cardiology. Therefore, in a masterpiece article, Timothy W. Churchill and Aaron L. Baggish address the most relevant aspects in the cardiovascular care of master athletes with focus on four of the most common clinical scenarios including atrial fibrillation, myocardial fibrosis, coronary artery disease and dilation of the ascending aorta [6].

The appearance of syncope is probably the most challenging and threating symptom in athletes, and it merits a specific article addressing its causes and the clinical work-up recommendations. Flavio D'Ascenzi et al. have written a fantastic review of this topic, with the inclusion of less known causes of syncope that are nonetheless not uncommon during the practice of extreme sports, such as apnoea-induced syncope during free scuba diving and the gravity-induced loss of consciousness in jet pilots [7].

Despite our efforts to identify athletes at risk of SCD and appropriate management recommendations, sudden cardiac arrest in the field of play will continue to occur. Commotio cordis, subclinical unknown forms of myocarditis among others, may trigger ventricular arrhythmias in otherwise healthy athletes, and we must be prepared to respond. The best preventive strategy on this scenario is the prompt recognition of the arrest and having on place a well-developed and known emergency action plan than can be rapidly activated ensuring early defibrillation, appropriate management of the athlete on the field of play with good transition care and safe transportation to the appropriate healthcare institution. Henry F Pelto and Jonathan A Drezner have excellently addressed this topic [8], guiding the reader on additional important considerations that must be included in the development of an appropriate emergency action plan such as specific sports considerations, specific venue considerations (open road/water event characteristics), extreme weather conditions and others.

While guidelines and recommendations in sports cardiology are important to guide the management of athletes with cardiovascular conditions, the final decision-making process in sports cardiology is always complex and must be individualized, especially in those cases falling in the wide grey zone of abnormal findings with inconclusive diagnostic assessment. Shared decision with the athlete, even shared consensus decision-athlete centred - including sometimes other involved parts such as athlete's family, team doctors, coaches or managers, may be required. In the last article of this special issue, three real practical situations are presented to illustrate the difficulty of sports cardiology decision-making process in which many factors must be considered including the athlete's will and preventive measures on place in the event of a major adverse cardiac event during sports participation. The clinical cases are brought to us by Zigor Madaria (case 1), Maria Dolores Masia (case 2), Maria Alcocer and Araceli Boraita (case 3), and other team members experienced sports cardiologists who highlight on their cases the important role of the cardiology follow-ups in athletes with cardiology conditions [9]. Indeed, management decisions evolve with the time, as the clinical profile changes with athlete's education on symptoms self-monitoring and regular cardiology assessment.

Therefore, this special issue compiles well-recognized and experienced authors, which have addressed relevant problems in outstanding review manuscripts that, with no doubt, will lead to spread and improve the clinical practice in the always appealing and challenging field of sports cardiology.

\section{References}

1. Abulí, M., de la Garza, M. S., \& Sitges, M. (2020). Differentiating athlete's heart from left ventricle cardiomyopathies. Journal of Cardiovascular Translational Research. https://doi.org/10.1007/ s12265-020-10021-8.

2. Zorzi, A., Cipriani, A., Mattesi, G., Vio, R., Bettella, N., \& Corrado, D. (2020). Arrhythmogenic cardiomyopathy and sports activity. Journal of Cardiovascular Translational Research. Springer. https://doi.org/10.1007/s12265-020-09995-2.

3. Vio, R., Zorzi, A., \& Corrado, D. (2020). Myocarditis in the athlete: arrhythmogenic substrates, clinical manifestations, management, and eligibility decisions. Journal of Cardiovascular Translational Research. Springer. https://doi.org/10.1007/s12265-020-09996-1.

4. Cardiovascular magnetic resonance and sport cardiology: a growing role in clinical dilemmas

5. Pieles, G. E., \& Oberhoffer, R. (2020). The assessment of the paediatric athlete. Journal of Cardiovascular Translational Research. https://doi.org/10.1007/s12265-020-10005-8.

6. Churchill, T. W., \& Baggish, A. L. (2020). Cardiovascular care of masters athletes. Journal of Cardiovascular Translational Research. Springer. https://doi.org/10.1007/s12265-020-09987-2.

7. D'Ascenzi, F., Zorzi, A., Sciaccaluga, C., Berrettini, U., Mondillo, S., \& Brignole, M. (2020). Syncope in the young adult and in the athlete: causes and clinical work-up to exclude a life-threatening cardiac disease. Journal of Cardiovascular Translational Research. Springer. https://doi.org/10.1007/s12265-020-09989-0.

8. Pelto, H. F., \& Drezner, J. A. (2020). Design and implementation of an emergency action plan for sudden cardiac arrest in sport. Journal of Cardiovascular Translational Research. Springer. https://doi.org/ 10.1007/s12265-020-09988-1.

9. The grey zone and the complexity of clinical decisions in sports cardiology

Publisher's Note Springer Nature remains neutral with regard to jurisdictional claims in published maps and institutional affiliations. 